\title{
On the Forward Scattering of Radio Waves in the Lower Ionosphere
}

\author{
Tor Hagfors

\begin{abstract}
Contribution from Norwegian Defence Research Establishment, Kjeller pr Lillestrøm, Norway.
\end{abstract}
(Received January 26, 1962; revised March 8, 1962)

\begin{abstract}
A method is developed whereby it is, in principle, possible to separate the two main signal components in VHF ionospheric forward scatter propagation. The method is based on a study of the angular power distribution of the received signal. From experiments made over a circuit of $1,180 \mathrm{~km}$ the law of turbulent scattering is determined. The spectrum for the electronic irregularities is found to be given by $k^{-n}$ with $n \cong 9$ under the experimental conditions described.
\end{abstract}

\section{Introduction}

It is by now nearly 10 years since the first results were published by Bailey et al., [1952] on that mode of propagation which has become known as VHF forward scatter. Since then a considerable amount of work has been done by many groups of research workers. These efforts have partly been designed to provide data for evaluation of the mode of propagation for communication purposes, and partly to provide an understanding of the basic propagation mechanisms involved. It is fairly evident that the usefulness of forward scattering in communication is rather limited. But people interested in ionospheric phenomena have found that in spite of this the mode of propagation has been well worth studying because of the interesting data which can be obtained on the irregular structure of the lower iono-sphere. In spite of this interest there does not seem to be universal agreement about all the details of the scattering mechanism as yet.

It is the purpose of the present paper to give a brief summary of certain aspects of some experimental work which was carried out in Norway prior to 1959 , and which has previously only been reported at length in publications not so easily a vailable [Hagfors, 1959]. The results to be described are concerned in the first place with the separation of the two main propagation modes and secondly with the study of the law of scattering of that part of the signal which is believed to be due to turbulent fluctuations in electron density.

In section 2 a brief review is given of the various theories that have been produced to explain the signal. Section 3 gives an account of the method of separating the two signal components. In Section 4 the experimental setup and the results of measurements are presented. Section 5 discusses the results and proposes a turbulent scattering law.

\section{Theories of the Scattering}

When the power from a VHF transmitter $\$ is beamed obliquely at the lower ionosphere, some of the power is scattered out of this beam and can be observed at a receiver at a distance of 1,000 to 2,000 $\mathrm{km}$. Because the frequency can be well above the MUF for the distance, there is general agreement that there must be some irregularities, blobs or steep gradients present to explain the deviation in direction of some of the wave. There are several theories on the origin of these irregularities. The two most widely accepted ones are those based on turbulence or on scattering from meteor trails. In the following, certain consequences of these theories are discussed.

\subsection{Scattering by Irregularities}

The random irregularities in electron density can be thought of as a superposition of structures of all sizes, and can be described by a spectrum of plane electron density waves $G(\vec{k})$ where the wavelength is $\frac{2 \pi}{\rightarrow}$ and where $\vec{k}$ is in the direction of the wave. $|k|$

The scattered power is determined by that component in the spectrum corresponding to $\vec{k}=\vec{k}_{1}-$ $\vec{k}_{2}$ where $\vec{k}_{1}$ and $\vec{k}_{2}$ are the wave-vectors of the incident and the scattered waves respectively. Let us put $\vec{k}_{1}-\vec{k}_{2}=\vec{K}$, the power received is then proportional to $G(\vec{K})$. Because $|K|=\frac{4 \pi}{\lambda} \cdot \sin (\theta / 2)$ where $\lambda$ is the radio wavelength and $\theta$ the angle through which the wave is scattered, we see that the scattering condi- 
tion is equivalent to the familiar Braggs condition for crystal scattering.

In Booker and Gordon's [1950] original paper on the subject a spatial correlation function was assumed, and the spectrum $G(\vec{K})$ derived from that. Villars and Weisskopf [1954] derived the spatial spectrum directly by methods which are known from the theories of homogeneous turbulence. In this first work electron density fluctuations were simply taken to follow the density fluctuations of the neutral gas. Later on this view was revised and a gradient in mean electron density introduced. Electron density fluctuations were then imagined to be produced by transport across this gradient [Villars and Weisskopf, 1955; Wheelon, 1957]. This model was later on criticized by Silverman [1956] and by Bolgiano [1958] who maintained that the gradients seen by the small structure would essentially be that created by the larger structure, and that the mean gradient would only aid in the production of the large irregularities.

It may safely be said that the situation regarding the theories of the origin and properties of the electron density fluctuations is in a rather unsatisfactory state. All that can be concluded at the moment appears to be that the scattering cross section can be approximated by an inverse power law in $|\vec{K}|$,

$$
G(\vec{K}) \sim|\vec{K}|^{-n}
$$

where $n$ may lie between 4 and 11 .

In recent studies of the variation of received power with frequency using scaled antennas it is often found that the power decreases with frequency as $f^{-m}$. The exponent $m$ is related to $n$ through $m-n=2$. A recent review of different scatter exponents has been given by Wheelon [1960].

\subsection{Scattering From Ionized Meteor Trails}

Meteoric particles penetrating into the earth's atmosphere leave behind a long straight trail. Most of the trails left behind by small meteoric particles disappear through a simple diffusion mechanism. Echoes from such trails build up in a matter of a few hundredths of a second and decay exponentially in a time of the order of seconds. The line density of these trails is less than about $10^{14}$ electrons per meter. Echoes from trails of greater density last longer and exhibit a much more irregular structure because the trails get time to become bent and twisted during the echo.

As long as the trails remain straight very strict reflection conditions apply for both types of ionized columns. The reflection point is found to be the point of tangency between the trail and one or other of a family of ellipsoids of revolution with transmitter and receiver as common foci. In addition, the reflection point must fall within the height range where the ionization is actually formed i.e., between about 100 and $120 \mathrm{~km}$. In practice the antenna systems are usually most sensitive to reflections near the midpoint of the path. Reflections at the great-circle plane near the midpoint of the path must be due to nearly horizontal trails. These can be shown to be very weakly ionized. Along the great circle path there is therefore a minimum of echoes and there are maxima at either side [Eshleman and Manning, 1954; Hines and Pugh, 1956].

Because the distribution of meteor radiants is a function of the time of day the distribution of echoes will be dependent on the time of day. For a NorthSouth path in northern temperate latitudes, a case of particular interest in the present study, reflections are symmetric about the great circle path at $6 \mathrm{a} . \mathrm{m}$. and at $6 \mathrm{p} . \mathrm{m}$. In the day time more reflections occur at the East side and by night more at the West side of this path.

Meteor echoes will, therefore, according to theory, always appear at either side of the great circle path between transmitter and receiver and during most of the time in an asymmetrical manner depending on the time of day and the time of the year. The meteor echoes will appear as short bursts of signal amplitude, and possibly as a continuous signal provided the signal bursts overlap.

\subsection{Discussion of Theories}

Other theories do exist, notably based on partial reflections from mean gradients [Feinstein and Salzberg, 1952] or on ionization associated with lightening discharges [Isted, 1954], but these are generally regarded as somewhat unlikely.

One of the important problems in connection with the understanding of the mode of propagation thus appears to be the separation of the turbulent scatter signal and the meteoric signal. The fairly strong meteor bursts which appear superimposed on the continuous background signal can quite easily be distinguished. But there must be large numbers of weak meteoric echoes hidden in the continuous signal. It might even be that the continuous signal at times consists of overlapping weak echoes. In the latter case it would not be possible to distinguish between turbulent scattering and meteoric reflections.

The property which is most distinctly different in the two types of continuous signals apparently lies in their distribution of power as a function of direction of arrival of the signal - or in the angular power spectrum. A turbulent scatter signal should have a maximum along the direction of the great circle path provided the turbulence is isotropic. A meteoric signal should exhibit a minimum along: the great circle path, and the shape of the angular spectrum should change systematically over the day.

In the present paper, use will be made of the difference in angular power spectra of a turbulent scatter signal and a meteor signal to distinguish between the two types of continuous signals. It will, in fact, be shown in the next section that a close study of the angular spectrum of the received signal is not only suitable in deciding which mechanism is active, but also to find their relative importance if both are simultaneously present. 


\section{Method of Observation}

We shall imagine that the geometry of the circuit to be considered can be represented as in figure 1 . Whenever necessary reference will be made to the actual circuit between Tromsö and Kjeller used in the experiments. In the next subsection a method is given for the determination of angular spectra by interferometer techniques, and certain calculations are made for various assumed turbulent scatter laws to determine what results to expect. In the following subsection a theory is given for the separation of a turbulent scatter component and a meteoric component.
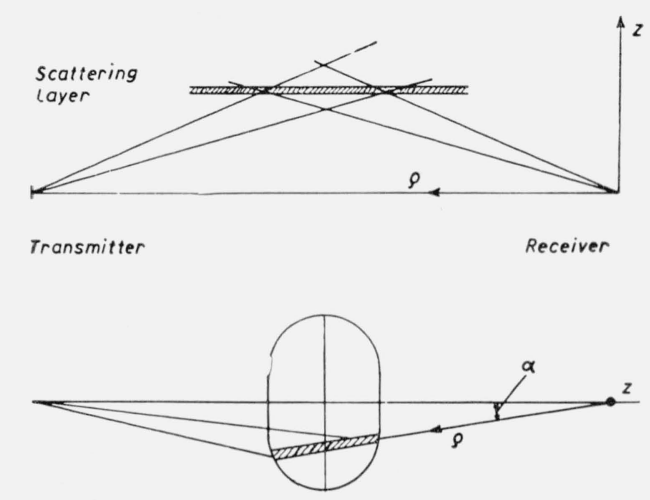

Figure 1. Geometry of the circuit.

\subsection{Principle of the Measurement of Angular Spectra}

Suppose the scattered wave field is viewed by two antennas displaced a distance $\xi$ wavelengths along a direction perpendicular to the transmitter receiver great circle path. At an instant $t$ the complex field from the direction specified by $\alpha=$ arc $\sin S$ at the midpoint of the aerial baseline is taken to be $F(S, t)$ $d S$. If there are many independent scattering elements within the total angular spectrum at any one time, the normalized complex correlation function $R(\xi)$ between the complex signals at the two antennas is given by:

$$
R(\xi)=\frac{\overline{e_{1} \cdot e_{2}{ }^{*}}}{\overline{\left|e_{1}\right|^{2}}}=\frac{\boldsymbol{S} \overline{\left.F(S, t)\right|^{2}} \exp (-2 \pi i \xi S) d S}{\mathcal{S} \overline{\left.F(S, t)\right|^{2}} d S} .
$$

Here $e_{1}$ and $e_{2}$ are the complex signals at the two antennas, the power in the two is assumed to be equal, star means complex conjugate and bar indicates time average. For convenience we henceforth put $\overline{\left.F(S, t)\right|^{2}}=|F(S)|^{2}$ which is the angular power spectrum. A Fourier relationship, therefore, connects the two quantities $|F(S)|^{2}$ and $R(\xi)$. A knowledge of $R(\xi)$ is thus a sufficient condition for the determination of $|F(S)|^{2}$.

Let us now turn to the problem of actually measuring $R(\xi)$ for various aerial spacings. Suppose the signals from the two aerials are combined through a four terminal network in such a way that for a plane wave incident along the great circle path (i.e., along $\alpha=0$ ) the two signal voltages are added in phase at one output terminal and in opposite phase at the other. The complex voltages at these two output terminals can in general be represented as:

$$
\begin{aligned}
& e_{\Sigma} \sim e_{1}+e_{2} \\
& e_{\Delta} \sim e_{1}-e_{2},
\end{aligned}
$$

no matter the type of angular spectrum of the incident wave field. The available powers at the two terminals are proportional to 'Re means real part):

$$
\begin{aligned}
& P_{\Sigma} \sim 2\left\{\overline{\left|e_{1}\right|^{2}}+\operatorname{Re} \overline{\left(e_{1} e_{2}^{*}\right)}\right\} \\
& P_{\Delta} \sim 2\left\{\overline{\left|e_{1}\right|^{2}}-\operatorname{Re} \overline{\left(e_{1} e_{2}^{*}\right)}\right\} .
\end{aligned}
$$

The correlation coefficient $\rho(\xi)$ is therefore found as:

$$
\rho(\xi)=\operatorname{Re}\left\{\frac{\overline{e_{1} e_{2} *}}{\overline{\left|e_{1}\right|^{2}}}\right\}=\frac{P_{\Sigma}-P_{\Delta}}{P_{\Sigma}+P_{\Delta}}=\frac{\mathcal{S}|F(S)|^{2} \cos 2 \pi \xi S d S}{\mathcal{S}|F(S)|^{2} \cdot d S} .
$$

From this correlation coefficient, which is the real part of $R(\xi)$, the angular power spectrum is uniquely determined only when $|F(S)|^{2}$ is symmetrical about $S=0$. For a scatter signal caused by isotropic irregularities alone, this condition is met. But since the continuous background signal we wish to study is a mixture of a turbulent component and a meteoric component, it might well happen that $\left|F\left(S^{\prime}\right)\right|^{2}$ is not symmetric. In practice it is fortunately easy to measure the imaginary part of $R(\xi)$ as well. Suppose the complex signal voltage $e_{1}$ is delayed by $90^{\circ}$ before entering our network. At the two output terminals the following voltages will now appear:

$$
\begin{aligned}
& \hat{e}_{\Sigma}=-i e_{1}+e_{2} \\
& \hat{e}_{\Delta}=-i e_{1}-e_{2},
\end{aligned}
$$

and the available power will be (Im means imaginary part):

$$
\begin{aligned}
& \hat{P}_{\Sigma} \sim 2\left\{\overline{\left.e_{1}\right|^{2}}+\operatorname{Im} \overline{\left(e_{1} e_{2}^{*}\right)}\right\} \\
& \hat{P}_{\Delta} \sim 2\left\{\overline{\left.e_{1}\right|^{2}}-\operatorname{Im} \overline{\left(e_{1} e_{2}^{*}\right)}\right\} .
\end{aligned}
$$

If these quantities are used formally as above to calculate a correlation coefficient denoted by $\hat{\rho}(\xi)$ one obtains:

$$
\hat{\rho}(\xi)=\operatorname{Im}\left\{\frac{\overline{e_{1} e_{2}{ }^{*}}}{\left|e_{1}\right|^{2}}\right\}=\frac{\hat{P}_{\Sigma}-\hat{P}_{\Delta}}{\hat{P}_{\Sigma}+\hat{P}_{\Delta}}=\frac{\mathcal{S}|F(S)|^{2} \sin 2 \pi \xi S d S}{\mathcal{S}|F(S)|^{2} d S},
$$


When both $\hat{\rho}(\xi)$ and $\rho(\xi)$ are known as functions of $\xi, R(\xi)$ is given by:

$$
R(\xi)=\rho(\xi)+i \hat{\rho}(\xi),
$$

and it is possible in a unique way to evaluate $|F(S)|^{2}$ whether the angular spectrum is symmetric or not.

For a turbulent scattering mechanism the angular spectrum is symmetric and $\hat{\rho}(\xi)$ is zero. The real
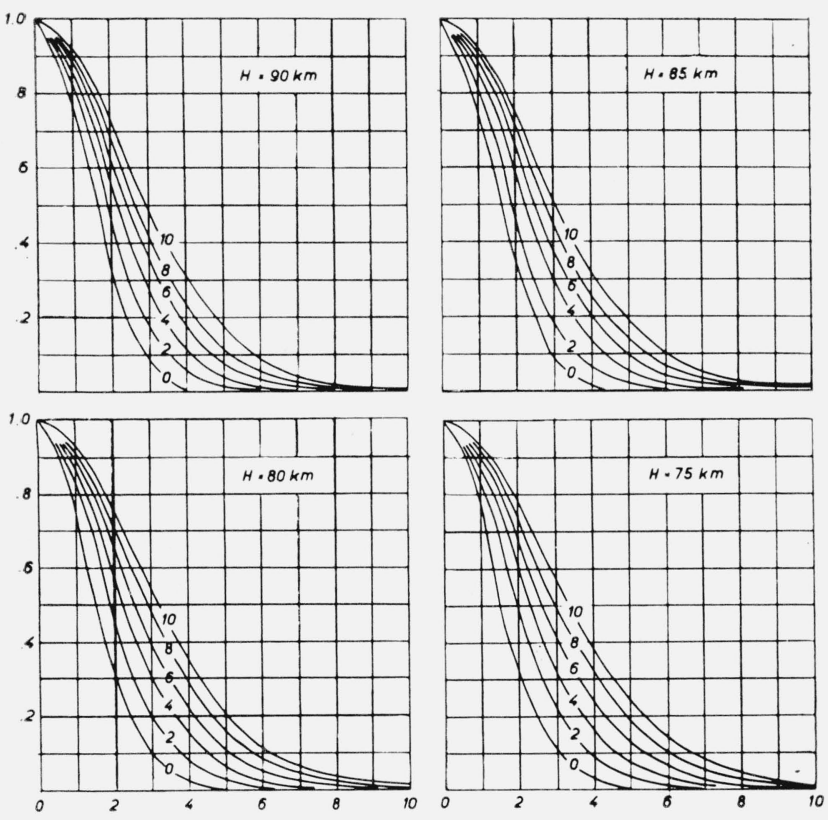

Aerial separation (wavelengths)

Figure 2. Correlation plotted against aerial spacing for various heights with scatter exponent as parameter.

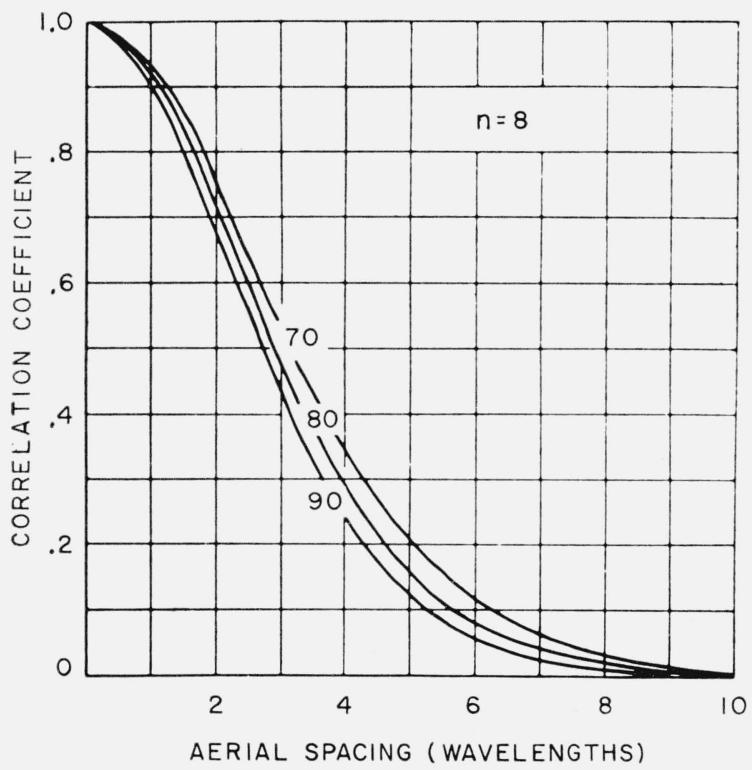

Figure 3. Correlation plotted against aerial spacing for $n=8$ with height as parameter. part of $R(\xi)$ was computed for various scatter exponents $n$ and for various assumed heights of scattering. Measured polar diagrams of the antennas were used in these calculations. Figure 2 gives a set of these correlation curves for various scattering heights with the exponent $n$ as parameter. The effect of height variation for a fixed scatter exponent $n=8$ is shown in figure 3 .

\subsection{Separation of Symmetric and Asymmetric Angular Spectra}

The method of separating two propagating modes which are simultaneously present is based on two assumptions:

(a) The angular spectrum produced by one of the propagation mechanisms is symmetrical about the transmitter receiver great circle path. This would be the case for a turbulent scatter signal if the turbulence were isotropic.

(b) The angular spectrum of the other propagation mechanism is unsymmetrical about the great circle path, and the form of this spectrum is known. Meteor reflections would generally produce such a spectrum during most of the day. Knowledge about the form of this spectrum can be obtained by a study of the distinct meteor bursts if it is assumed that the indistinguishable meteor bursts have the same angular spectrum as the distinct ones.

With two independent propagation mechanisms present simultaneously the total angular power spectrum, $|F(S)|^{2}$, and the total power, $P$, may be split into two parts, one due to turbulent scattering (index " $s ")$, and the other due to meteor reflections (index " $m$ "):

and

$$
|F(S)|^{2}=\left|F_{s}(S)\right|^{2}+\left|F_{m}(S)\right|^{2}
$$

$$
P=P_{s}+P_{m}
$$

There is a relation between these quantities:

$$
\begin{aligned}
P & =\mathcal{S}|F(S)|^{2} d S \\
P_{s} & =\mathcal{J}\left|F_{s}(S)\right|^{2} d S \\
P_{m} & =\mathcal{J}\left|F_{m}(S)\right|^{2} d S .
\end{aligned}
$$

The complex correlation coefficient $R(\xi)$ of the total background signal can, by simple manipulations, be expressed by the complex correlation coefficient of the turbulent scatter signal, $R_{s}(\xi)$, and by that of the meteoric contribution, $R_{m}(S)$ in the following way:

$$
R(\xi)=\frac{P_{s}}{P} \cdot R_{s}(\xi)+\frac{P_{m}}{P} \cdot R_{m}(\xi) .
$$

Since $R(\xi)$ can be expressed by its real and imaginary parts, which can both be measured, one obtains the two equations:

$$
\rho(\xi)=\frac{P_{s}}{P} \rho_{s}(\xi)+\frac{P_{m}}{P} \cdot \rho_{m}(\xi)
$$




$$
\hat{\rho}(\xi)=\frac{P_{s}}{P} \hat{\rho}_{s}(\xi)+\frac{P_{m}}{P} \hat{\rho}_{m}(\xi)
$$

Application of condition (a) above implies, however, that $\left|F_{s}(S)\right|^{2}$ is symmetric and that $\hat{\rho}_{s}(\xi)=0$. Hence:

$$
\hat{\rho}(\xi)=\frac{P_{m}}{P} \cdot \hat{\rho}_{m}(\xi)
$$

and

$$
\rho(\xi)=\frac{P_{s}}{P} \rho_{s}(\xi)+\hat{\rho} \cdot \frac{\rho_{m}(\xi)}{\hat{\rho}_{m}(\xi)} .
$$

By the simple substitution:

$$
\frac{P_{s}}{P}=1-\frac{P_{m}}{P}=1-\frac{\hat{\rho}(\xi)}{\hat{\rho}_{m}(\xi)}
$$

one finally finds for the correlation coefficient of the turbulent scatter signal alone:

$$
\rho_{s}(\xi)=\frac{\rho(\xi) \hat{\rho}_{m}(\xi)-\hat{\rho}(\xi) \cdot \rho_{m}(\xi)}{\hat{\rho}_{m}(\xi)-\hat{\rho}(\xi)} .
$$

The evaluation of the correlation coefficient of the turbulent scattering alone therefore requires that both $\rho(\xi)$ and $\hat{\rho}(\xi)$ are measured, and that the shape of the angular distribution of distinct meteoric reflections is known. Application of condition (b) above implies that the correlation coefficients $\rho_{m}(\xi)$ and $\hat{\rho}_{m}(\xi)$ found for distinct meteor bursts are the same as for those hidden in the background signal.

Various power ratios of interest can easily be deduced and are listed below:

$$
\frac{P_{s}}{P}=1-\frac{\hat{\rho}(\xi)}{\hat{\rho}_{m}(\xi)}, \quad \frac{P_{m}}{P_{s}}=\frac{\hat{\rho}(\xi)}{\hat{\rho}_{m}(\xi)-\hat{\rho}(\xi)}, \quad \frac{P_{m}}{P}=\frac{\hat{\rho}(\xi)}{\hat{\rho}_{m}(\xi)} .
$$

The above analysis thus affords a means whereby the relative importance of the two mechanisms can be assessed and whereby the turbulent scatter component can be extracted from the total background signal and studied separately.

\section{Observations}

This section gives a description of the equipment used in the analysis of angular power spectra of signals received over a test circuit between Tromsö $\left(70^{\circ} \mathrm{N}, 19^{\circ} \mathrm{E}\right)$ and Kjeller $\left(60^{\circ} \mathrm{N}, 11^{\circ} \mathrm{E}\right)$. Results of a series of tests with this equipment are summarized in the following subsection.

\subsection{Equipment}

The transmitter of approximately $5 \mathrm{kw}$ continuous wave at $46.8 \mathrm{Mc} / \mathrm{s}$ near Tromsö feeds into a small rhombic aerial whose polar diagram is known from measurements. At Kjeller four 6-element yagi aerials were erected along a line perpendicular to the Tromsö-Kjeller great circle path providing the following aerial spacings when combined in pairs: $2,3,4,5,7$, and 9 wavelengths. The polar diagrams of the yagi aerials are likewise known from measurements. The signal received after passing a balun transformer were fed through coaxial cables of identical length to the receiving equipment. Here the received signals were combined in pairs in a hybrid ring ("rat-race"). If the signals at the two input terminals are identical both in phase and amplitude, the available power will be zero at the $\Delta$ output terminal, and equal to the total power fed into the circuit at the $\Sigma$ output terminal. A plane wave incident perpendicular to the aerial base line, on account of identical aerials and cables, produces the full power from two aerials at the $\Sigma$ terminal and zero power at the $\Delta$ terminal. Plane waves incident at an angle $\alpha$ with the normal to the aerial baseline will produce at the two output terminals powers proportional to $\cos ^{2}\left(\frac{\pi a}{\lambda} \sin \alpha\right)$ and $\sin ^{2}\left(\frac{\pi a}{\lambda} \sin \alpha\right)$ respectively, $a$ being the aerial spacing. The outputs from the hybrid ring are fed to separate cascode preamplifiers, separate receivers $(\mathrm{R} \mathrm{X})$, detectors and amplifiers (Det), on to a twin channel pen recorder, with special amplifier. The lining up of the whole interferometer system, which is evidently very critical, was controlled by a test transmitter located in front of the receiving aerials at a distance of about $2 \mathrm{~km}$.

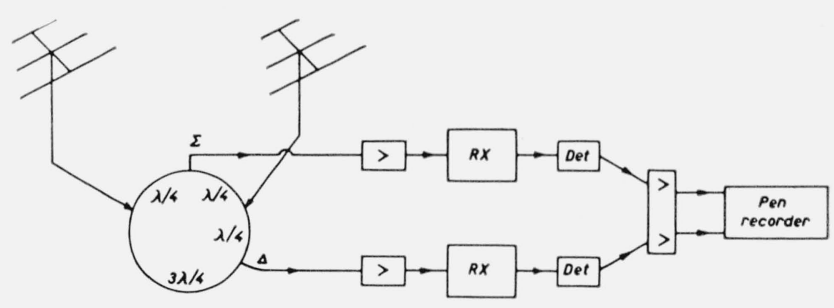

Figure 4. Receiving system.

The delay by $90^{\circ}$ of one of the signals, which is required to obtain the sine transform of the angular power spectrum, was effected by inserting a cable of that electrical length between one of the aerial cables and the hybrid circuit.

The routine calibration of the two channels was made as follows. A signal from a signal generator was applied to one of the hybrid input terminals. By noting the signal generator attenuator reading and the pen deflections at the different signal levels a calibration curve could be obtained for both channels simultaneously. Routine calibrations were made at least once during a half hour observation. If drift was suspected, calibrations were made before and after the observation and the record was rejected if the drift was appreciable. 


\subsection{Observational Results}

The observations described here were taken between October 1957 and August 1958. The transmitter was switched on every hour and off half past every hour. During a half hour interval samples of approximately 2 min duration were taken for each aerial separation, most of the samples at the small aerial spacings since these were considered to be of greatest interest. It should be noted that observations were only made when a continuous background signal was present well above the noise level.

In analyzing the samples, all clear meteor bursts were removed from the record. Then the median signal level was found for the $\Sigma$ and the $\Delta$ channels. The power at the two output terminals was taken to be proportional to the square of the median level This is justified by the fact that the background signal appears to be distributed in the same way as band pass limited random Gaussian noise.

Correlation coefficients were determined from formulae (3) and (5) developed in the previous section. The number of samples analyzed for the different aerial spacings are given in table 1.

TABLE 1. Number of samples analysed

\begin{tabular}{l|r|r|r|r|r|r}
\hline \hline Spacing wavelengths_...... & 2 & 3 & 4 & 5 & 7 & 9 \\
Number $\rho(\xi) \ldots \ldots$ & 59 & 57 & 50 & 27 & 15 & 7 \\
Number $\hat{\rho}(\xi) \ldots \ldots \ldots \ldots$ & 41 & 42 & 15 & 2 & 1 & 0
\end{tabular}
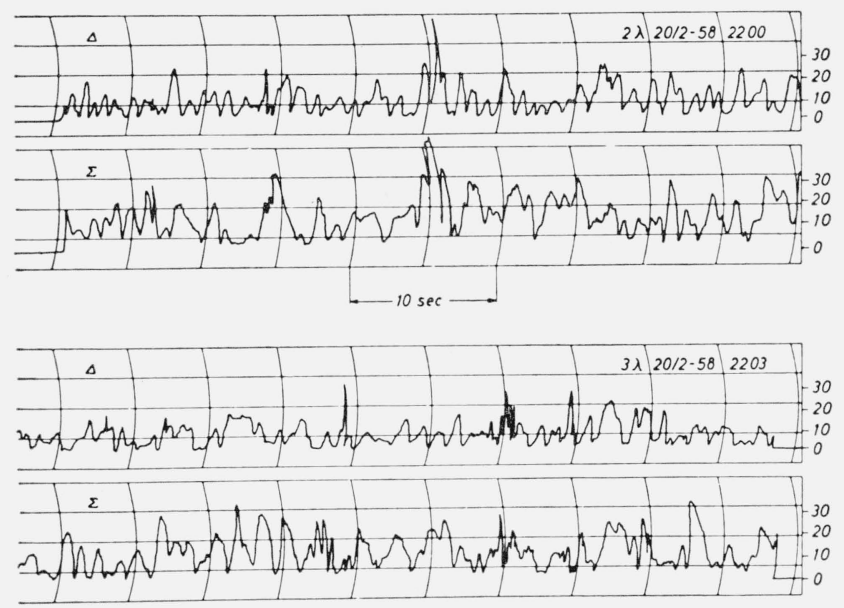

Figure 5. Examples of interferometer records.

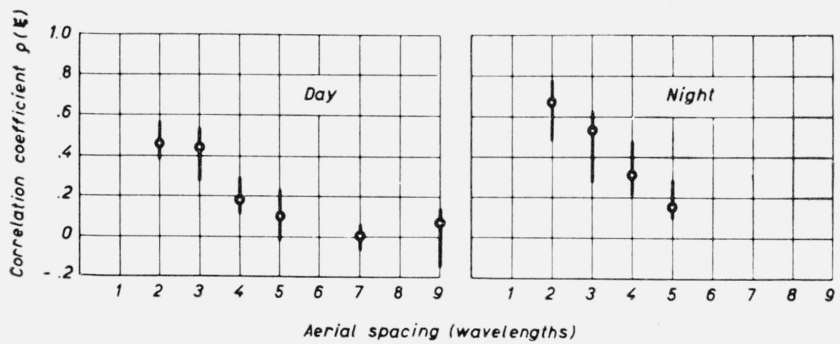

Figure 7. Median and interquartile range of correlation coefficients, day and night values separated.
Two examples of interferometer records are shown in figure 5 .

The median values of the correlation coefficients for the different aerial spacings together with upper and lower quartiles are plotted in figure 6. Perhaps the most striking feature about this plot is the relatively low value of $\rho(\xi)$ found at a spacing of 2 wavelengths.

It is now worth while inquiring in more detail whether there is a dependence of the data on the time of day or on signal level. To this end the data were divided in day-values, comprising all values obtained between 0900 and $1430 \mathrm{LT}$, and night data including all values obtained between 2100 and 0230 L'T. For these two groups of data the medians and the interquartile range were again plotted against spacing, see figure 7 .

It is seen that there is a difference between the two sets of data in that the night values are generally higher than those obtained during daytime. Next let us turn to the asymmetric component of the angular power spectrum, represented by the correlation coefficient $\hat{\rho}(\xi)$. For reasons which will become apparent from the next section it is again advantageous to separate the data into two groups, one including night values and the other day values as specified above. The medians of the two groups of data are plotted together with interquartile ranges in figure 8 . It is seen from these plots that the signal energy appears to come from the East side of the great circle path by day and, less

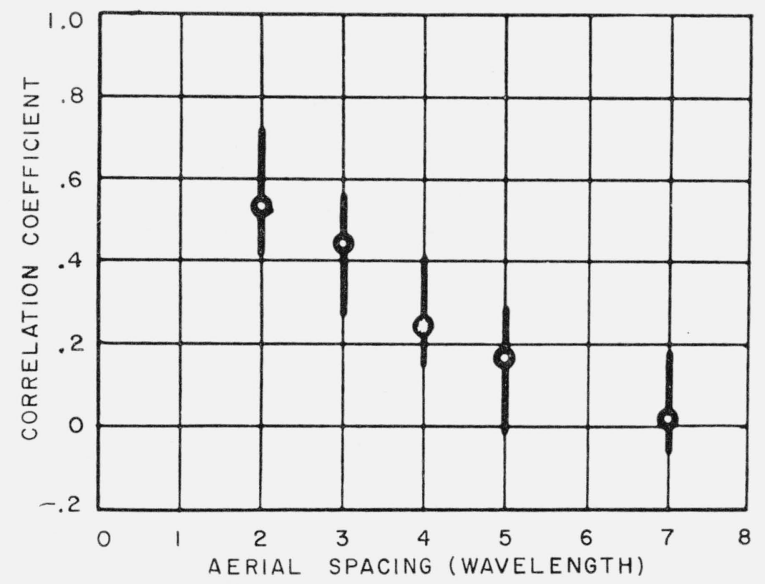

FIGURE 6. Median and interquartile range of correlation coefficients plotted against aerial spacing.

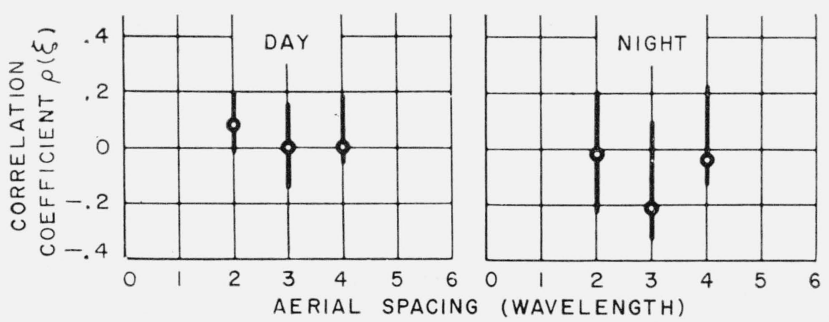

FIGURE 8. Demonstration of asymmetry in the direction of arrival of the received signal. 
significantly, from the West side of that path by night.

This effect is only apparent for the two smallest serial spacings, and not even for these are the results statistically significant. To test whether the correla-. coefficients $\rho(\xi)$ are in any way dependent on the signal level, a mass plot was prepared of the corre lation coefficients against received signal power $\left(P_{\Sigma}+P_{\Delta}\right)$ for the two smallest aerial spacings. Fig ure 9 in fact shows that there is an increase of the correlation coefficients with signal power, and that this is more clear for $\rho(2)$ than for $\rho(3)$.
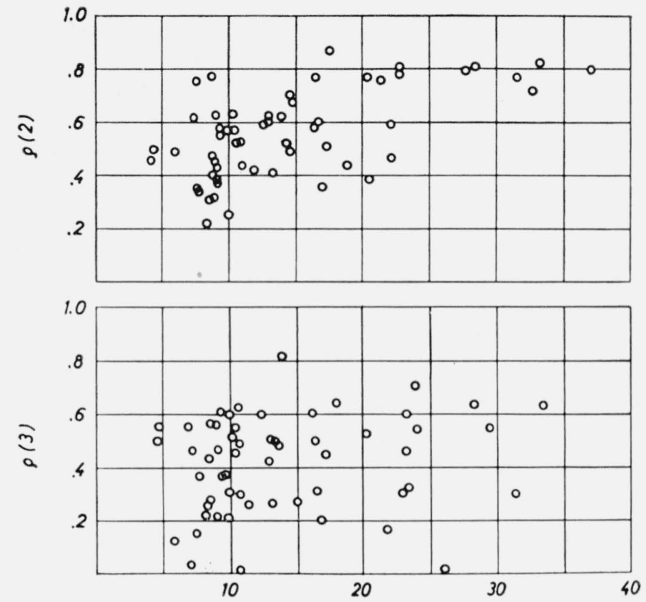

Figure 9. Plot of correlation coefficients against received signal power.

\section{Interpretation and Discussion}

An attempt will now be made to discuss the observational results of the previous section in the light of present knowledge on the problem of ionospheric forward scatter propagation. The results above, are clearly insufficient for the formation of a consistent picture of the mode of propagation on their own, but together with previous results of others, and of the author, it will be shown that a reasonable explanation of ionospheric forward scattering may be found.

\subsection{Scatter Law}

The dependence of the correlation coefficients on aerial spacing found from observations on the continuous background signal does not follow a law of the type assumed in the calculations of section 3. From the shape of the observed $\rho(\xi)$ curves, in particular the low median value at the smallest aerial spacing, one is lead to conclude that the power received from off path directions is, on the average, considerably higher than expected from any scatter law hypothesis.

To see whether this discrepancy means that the background signal is caused by overlapping meteor bursts, it is required to know the angular spectrum and the correlation coefficients of a meteoric signal. This information can be obtained in several ways.
From previous work by K. Endresen et al., [1958] the number of meteoric bursts received within various (azimuth) angular intervals is known as a function of time of day for the Tromsö-Kjeller test circuit. The minimum in the number of bursts seen along the great circle path, as predicted by theory, here received experimental verification. It was also shown how, due to the rotation of the earth relative to the apex of the earth's path, the angular distributions of number of bursts were systematically asymmetrical according to the time of day, so that round noon most bursts appeared on the East side of the great circle path and round midnight on the West side of this path. The noon and midnight mean angular distributions are shown in figure 10 . It might seem questionable to identify the angular number distribution with the angular power distribution in particular because the duration of the bursts is related to the azimuth angle of arrival. The effect of correcting for the duration was not very marked, as can be seen from figure 11.

Comparing the data of figure 11 with the observed correlation coefficient (fig. 6) it is seen that overlapping meteor bursts cannot possibly account for the signal. No single one of the correlation curves observed decrease as rapidly as the ones to be expected from overlapping meteor bursts alone. This mechanism must therefore be discarded as an explanation of the continuous background signal, at least the background signal seen with the sensitivity of our equipment.

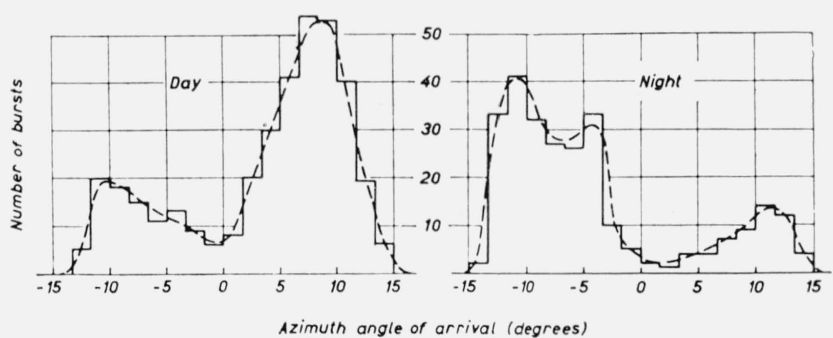

Figure 10. Angular distributions of number of burst. - . - smoothed distribution.

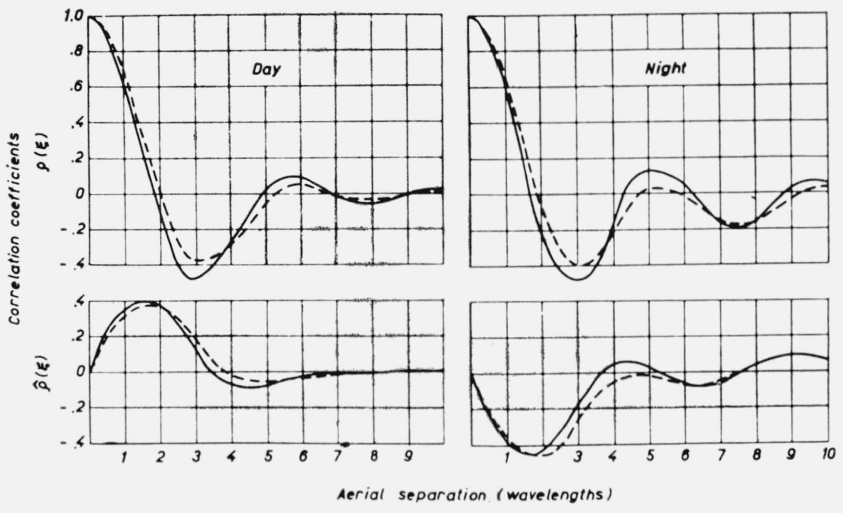

FIgure 11. Complex correlation coefficients $\mathrm{R}(\zeta)=\rho(\xi)=\mathrm{i} \rho(\zeta)$ expected from a purely meteoric signal. 
Next the question may be raised if the observed results could be explained in terms of a changing exponent due to the cut off wavenumber $k_{s}$, lying within the range of wavenumbers involved in the experiment. Turbulence theory predicts an increase in the effective exponent $n$ with $k$ under these circumstances [Batchelor, 1956]. This would cause the correlation coefficient to increase at low aerial separations, again incompatible with the observations.

Now let us try to explain the variation in received signal power in terms of a variation in the mechanical power supplied to the turbulent velocity field, and let us imagine the mean electron density to remain constant. An increase in the turbulent power supply may be imagined to cause more violent motions and hence greater deviations from the mean of electron density with stronger scattering. But, again, according to current ideas about homogeneous turbulence, an increase in power supplied will cause the cutoff wavenumber $k_{s}$ to increase [Batchelor, 1956] as $\epsilon^{\frac{1}{4}}$ ( $\epsilon$ being the power supplied) with the result that the effective exponent should decrease. The observed exponent is in fact found to increase with increasing received power. It appears that the observed results cannot be explained either by meteors alone or by a turbulent scatter mechanism alone. It is difficult, therefore, to avoid the conclusion that the continuous background signal is a mixture of a component due to turbulent scattering, and another due to meteor bursts which cannot be distinguished from the total signal.

On this assumption it is interesting to try an explanation of the observational results. The relatively low value of the correlation coefficient at the smallest aerial spacings may then be easily understood. Any meteoric contribution present together with the turbulent component will tend to reduce the correlation coefficients, and particularly at low aerial spacings, see formula (7) and figure 11.

The increase in correlation coefficients with increasing signal power can be ascribed to the increase of the turbulent scatter signal in relation to the meteoric component. This may not be readily seen since an increase in the background signal level will gradually conceal an increasing number of meteoric bursts. An approximate calculation is, therefore, made of the relative power of the meteors and of the background signal.

Suppose all the meteor bursts are of the exponentially decaying type with the same time constant $\tau$. The energy contained in a single burst of peak amplitude $a$ is then given by (power in unit resistance) :

$$
\frac{a^{2}}{2} \tau
$$

The number of bursts per second with peak amplitude in the range $a, a+d a$ is assumed to be given by $\nu(a) d a$ where

$$
\nu(a) \sim a^{-(s+1)}
$$

with $s$ somewhere between 1 and 1.5 [Hagfors, 1957].
Let us arbitrarily select as a criterion of an indistinguishable burst that its peak amplitude is smaller than the median signal level. If the same distribution law applies for the small as for the large meteor bursts, the power due to meteors in the background signal is given by:

$$
P_{m}=\text { const. } \frac{\tau}{2} \int_{0}^{a_{\mathrm{med}}} a^{2} \cdot a^{-(s+1)} d a \sim a_{\mathrm{med}}^{2-s}
$$

The power in the meteoric contribution is therefore at most proportional to the median signal level $a_{\text {med, }}$ whereas the scatter contribution is proportional to $a_{\text {med }}{ }^{2}$. The relative importance of meteors in the background signal is thus decreasing with increasing continuous signal power as required.

From the discussion so far, it appears that the best one can do to deduce the law of turbulent scattering is to select the correlation data obtained during strong signal conditions, and, in view of the limited amount of data, regardless of the time of day. The criterion of a strong signal was chosen to be a signal exceeding a total received power equivalent to $15 \mu$ from the signal generator used for calibration. The number of correlation coefficients selected in this way is given as a function of aerial spacing in table 2. The median values found and the interquartile ranges are plotted in figure 12 together with theoretical curves computed for a height of scattering of $85 \mathrm{~km}$, which has been found by others to be representative [Pineo, 1956].

It is seen that these median values follow a law of the type expected, and that the effective exponent $n$ lies somewhere between 8 and 10 .

The high effective scatter exponent can only be understood from previous theoretical work provided

\begin{tabular}{|c|c|c|c|c|c|c|}
\hline$\xi$ & 2 & 3 & 4 & 5 & 7 & 9 \\
\hline$N_{-}$ & 19 & 19 & 15 & 11 & 7 & 3 \\
\hline
\end{tabular}
the scales of the irregularities involved in this experi-

TABLE 2. Number of strong signal correlation coefficients

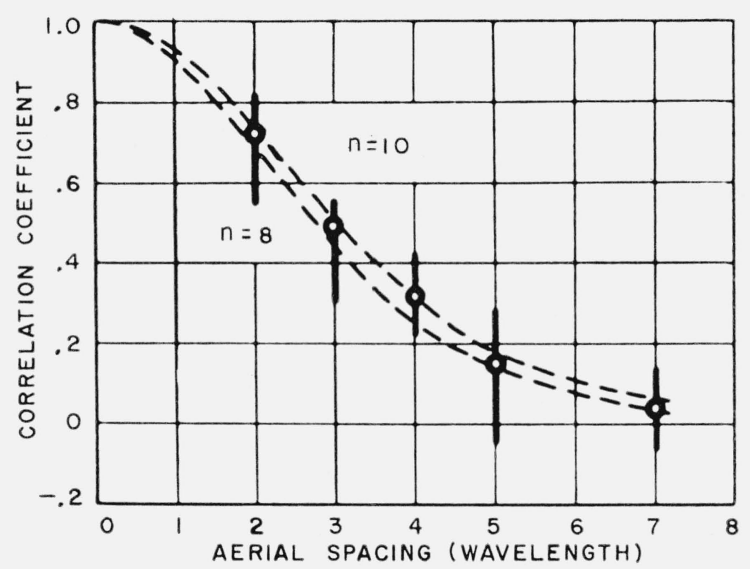

FIgure 12. Correlation coefficients during strong signal conditions. 
ment are all within the dissipation range of the turbulent velocity spectrum. 'This means that the scale corresponding to the transition wave number $k_{s}$ must be of the order of $2.8 \mathrm{~m}$ or larger. An exponent 9 has, in fact, according to Booker and Cohen [1956] been found both by R. A. Silverman and by G. K. Batchelor based on a turbulent mixing theory. The result found may, therefore, be regarded as in agreement with theory on the above condition.

The conclusion reached here should be compared with experimental results of others. The most serious attempts to determine the law of scattering has been made at NBS. Two types of experiments were made to evaluate the scatter exponent, both of which depend on measuring absolute values of transmission loss very accurately.

In the first type of these experiments the transmission loss was measured on scaled aerials at several frequencies between 30 and $108 \mathrm{Mc} / \mathrm{s}$, Blair [1959] and Blair, Davis, and Kirby [1961]. The general conclusion to be drawn from these experiments is that the scattering exponent is in the range 6 to 7 rather than 9 as found in the present work. The reason for the higher exponent found here may be as follows: the signals received in the frequency law experiment are to some extent influenced by a meteoric contribution. It is known that the power received by meteor reflections is less dependent on frequency than that of the continuous signal. The relative contribution to the received signal from meteors will, therefore, increase with frequency and the value of $n$ determined from a frequency law experiment will be too low.

The consistent inverse power frequency dependence through a wide frequency range found in these experiments seems difficult to explain from the picture developed in this paper.

The second type of experiment is the comparison of on-path and off-path signals. V. C. Pineo [1957] has conducted experiments to determine the ratio of powers received in the off-path and on-path circuits at $49 \mathrm{Mc} / \mathrm{s}$ and thus determine the scatter law. Because little was done to eliminate the effect of the meteors the exponent $n$ can be expected to be lower than that of the turbulent scattering alone. This appears to be the case. Pineo finds a value of about 6 .

The value of $n$ found in the present work is therefore thought to be quite reasonable in view of experimental results obtained elsewhere.

\section{Conclusion}

From the foregoing discussion it appears that ionospheric forward scattering at $\mathrm{VHF}$ can be well explained by two different propagation modes simultaneously present, one due to reflections from ionized meteor trails at a height of about $100 \mathrm{~km}$, and the other due to scattering from electronic irregularities caused by turbulence in the neutral air at about 80 to $85 \mathrm{~km}$. The continuous signal normally encountered is mainly due to turbulent scattering, and the meteoric power contaminating the continuous signal is confined to discreet though indistinguishable bursts. Any continuous signal present caused by overlapping meteor bursts must be well below the continuous signal normally originating from turbulent scattering and the spectrum of electronic irregularities in the wave number region above $0.35 \mathrm{~m}^{-1}$ is given by:

$$
G(k) \sim k^{-9}
$$

which probably means that this range of $k$ values are within the "dissipation region" of the turbulent velocity spectrum. The constant of proportionality has deliberately been omitted since its determination depends on a knowledge of absolute transmission loss as well as knowledge about the variation with height of the scattering. Little information on the latter question is available, though it appears that small scale turbulence is not well developed at 100 $\mathrm{km}$, but is present at $85 \mathrm{~km}$.

The methods of investigation developed in this paper is thought to be quite powerful, and similar types of experiments may well be extended to a detailed study of the diurnal and seasonal variation of the scattering and hence of the turbulent motion in the lower ionosphere.

The work described froms part of a research project carried out under the sponsorship of the Mutual Weapons Development Program under contract number $\mathrm{N}-01-\mathrm{MWP}-\mathrm{AF}-56$. The author is indebted to Mr. F. Lied and Dr. B. Landmark and to other colleagues at N.D.R.E. for suggestions, discussions, help, and advice.

\section{References}

Bailey, D. K., R. Bateman, L. V. Berkner, et al., A new kind of radio propagation at very high frequencies observable over long distances, Phys. Rev. 86, 141 (April 1952).

Batchelor, G. K., The theory of homogeneous turbulence (Cambridge University Press, Cambridge, 1956).

Blair, J. C., Frequency dependence of VHF ionospheric scattering, unpublished report (1959).

Blair, J. C., R. M. Davis, Jr., and R. C. Kirby, Frequency dependence of D-region scattering at VHF, J. Research NBS 65D (Radio Prop.), 417 (Sept.-Oct. 1961).

Booker, H. G., and W. E. Gordon, A theory of radio scattering in the troposphere, Proc. I.R.E. 38, 401 (April 1950).

Booker, H. G., and R. Cohen, A theory of long-duration meteor echoes based on atmospheric turbulence with experimental confirmation, J. Geophys. Research 61, 707 (Dec. 1956).

Bolgiano, R., The role of turbulent mixing in scatter propagation, Trans. I.R.E. AP-6, 161 (April 1958).

Endresen, K., T. Hagfors, B. Landmark, et al., Observations of angle of arrival of meteor echoes in v.h.f. forward scattered propagation, J. Atmospheric and Terrest. Phys. 12, 320 (1958).

Eshleman, V. R., and L. A. Manning, Radio communication by scattering from meteoric ionization, Proc. I.R.E. 4\%, 530 (March 1954).

Feinstein, J., and C. Salzberg, Ionospheric reflection of VHF radio waves, unpublished report (1952).

Hagfors, T., Some statistical results on reflection from meteor trails obtained by forward scatter experiments in Norway, AGARDograph $\mathbf{2 6}, 27$ (1957). 
Hagfors, T., Investigation of the scattering of radio waves at metric wavelengths in the lower ionosphere, Geophysica Norvegica 11, No. 2 (Aug. 1959).

Hines, C. O., and R. E. Pugh, The spatial distribution of signal sources in meteoric forward-scattering, Can. J. Phys. 34, 1005 (Oct. 1956).

Isted, G. A., Atmospheric electricity and long distance very high frequency scatter transmissions, Marconi Rev. 1\%, 37 (1954).

Pineo, V. C., Oblique-incidence measurements of the heights at which ionospheric scattering of VHF radio waves occurs, J. Geophys. Research 61, 165 (June 1956).

Pineo, V. C., Experimental observations of the contribution of meteoric ionization to the propagation of $\mathrm{VHF}$ radio waves by ionospheric forward scatter, unpublished report (1957).
Silverman, R. A., Turbulent mixing theory applied to radio scattering, J. Appl. Phys. 27, 699 (July 1956).

Villars, F., and V. F. Weisskopf, The scattering of electromagnetic waves by turbulent atmospheric fluctuations, Phys. Rev. 94, 232 (April 1954).

Villars, F., and V. F. Weisskopf, On the scattering of radio waves by turbulent fluctuations of the atmosphere, Proc. I.R.E. 43, 1232 (Oct. 1955).

Wheelon, A. D., Radio frequency and scattering angle dependence of ionospheric scatter propagation at $\mathrm{VHF}$, J. Geophys. Research 62, 93 (Jan. 1957).

Wheelon, A. D., Relation of turbulence theory to ionospheric forward scatter propagation experiments, J. Research NBS 64D (Radio Prop.) 301 (1960).

(Paper 66D4-204) 